\title{
Using Equation-Free Macroscopic Analysis for Studying Self-Organising Emergent Solutions
}

\author{
Giovanni Samaey, Tom Holvoet, Tom De Wolf \\ Department of Computer Science \\ Katholieke Universiteit Leuven, Belgium \\ Giovanni.Samaey,Tom.Holvoet@cs.kuleuven.be
}

\begin{abstract}
When engineering distributed software systems based on self-organising emergent solutions, assessing and understanding the relation between the microscopic dynamics and the resulting macroscopic behaviour is a fundamental issue. In our research, we investigate a systematic approach for understanding the link between microscopic and macroscopic behaviour, based on a numerical analysis technique called Equation-Free Macroscopic Analysis. Instead of deriving a (simplified) macroscopic model, equation-free methods only assume that such a model exists, and mimic a macroscopic simulation using only appropriately initialised simulations with the complete system.

There are two crucial issues in this technique. The first issue is defining a complete set of macroscopic variables that would uniquely characterise the self-organising emergent behaviour in the solution. The second issue is the definition of a suitable initialisation operator, that can create a good initial condition for the complete system, given only the values of the macroscopic variables.

In this paper, we propose a bottom-up approach for the selection of macroscopic variables and the related initialisation operator. We show how the equation-free approach can guide simulations to systematically increase understanding of the studied system. To illustrate the approach, we use a data clustering system inspired by termite nest building algorithms.
\end{abstract}

\section{Introduction}

Engineering software systems based on self-organising emergent solutions, where macroscopic system behaviour emerges from microscopic actions and interactions, is a difficult task. Mostly ad hoc approaches are used in proposing and evaluating such solutions, yielding superficial claims and directives about system wide behaviour over time - no guarantees can be provided. This is one acknowledged reason why such solutions are hard to infiltrate in real industrial settings.

In our research, we investigate a systematic approach for understanding the link between microscopic an macroscopic behaviour. We embed this approach in a de facto standard software engineering process - the Universal Process (UP) - and identify the activities in this process where a more rigourous approach to developing self-organising systems fits in. In fact, proposing a self-organising emergent solution corresponds to an architectural choice which a software engineer puts forward for accomplishing the functional as well as the non-functional (or quality) requirements. Activities in architectural design entail both identifying the necessary components, their relationships and their behaviour, and evaluating the proposed solution against the requirements.

Evaluating a self-organising emergent solution should aim to understand the solution and to make qualified statements upon the system-wide behaviour. In purely theoretical settings, where mathematical models are available, such evaluation can be founded on mathematical analysis techniques and tools. However, for real-world industrial applications that are 'engineered', mathematical models are typically not available. For example, decentralised control software for managing automated guided vehicles in a warehouse environment [30] is a complex system in terms of software and hardware - mathematical models are not available for such systems. Simulations can bring insight in the system-wide behaviour, yet cannot provide well-founded guarantees about the system-wide behaviour.

A promising "middle way", that bridges simulationbased and model-based approaches, was recently proposed in the numerical analysis literature. With this methodology, which has been called "equation-free macroscopic analysis", instead of deriving a (simplified) macroscopic model for a given self-organising system, one only assumes that such a model exists. One then constructs a so-called "coarse time-stepper" to mimic a simulation with the (unavailable) 


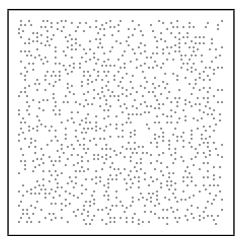

(a) start

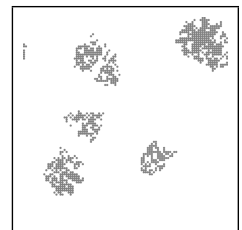

(b) after 1000 steps

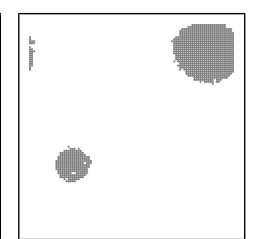

(c) after 9000 steps
Figure 1. Data Clustering case.

macroscopic model, using only appropriately initialised short simulations with the complete system. Since this approach appears to combine the advantages of simulationbased and model-based approaches for self-organizing solutions, we are currently investigating how, and to what extent, this methodology can be applied in this context. This paper presents the results of the study of this technique in engineering practical, non-trivial applications [8].

When applying this technique, there are two crucial step. First, one needs to define a set of macroscopic variables that completely characterise the self-organising emergent behaviour in the solution; these would be the variables that would appear in a macroscopic model. Second, one needs to construct a suitable initialisation operator, that can create a good initial condition for the complete system, given only the values of the macroscopic variables.

In this paper, we propose a bottom-up approach for the selection of macroscopic variables and the related initialisation operator. To contrast with pure brute force simulation, we show how this approach uses simulations with the full system to systematically refine understanding of the factors that influence macroscopic evolution. As an illustration, we use a data clustering system inspired by termite nest building algorithms.

The paper is structured as follows. Section 2 describes the data clustering case study that we use to illustrate our approach. Then, Section 3 provides an overview of equationfree macroscopic analysis. Section 4 clarifies the fact that identifying macroscopic variables and constructing an initialisation operator are crucial issues in the equation-free analysis technique, which can be used to systematically and iteratively build an understanding of the microscopic and macroscopic behaviour. This is applied to the data clustering case study in Section 5, and discussed in Section 6. Related work is discussed in Section 7. The paper concludes with final remarks and remaining challenges in Section 8.

\section{Case Study: Data Clustering}

We motivated the research by referring to issues that arise for real industrial applications. We use a rather simple case here, however, to illustrate our research. In particular, we investigate a self-organising emergent solution for a data clustering problem. Data clustering is a powerful tool for discovering structure in data. Clustering is the unsupervised classification of patterns (observations, data items, or feature vectors) into groups (clusters) [16]. The clustering problem has been addressed in many contexts and by researchers in many disciplines; this reflects its broad appeal and usefulness as one of the steps in exploratory data analysis. We refer to [16] for a good review on classical clustering algorithms.

Classical algorithms are static, centralised and batch [22]. They are static because they assume that the data and similarity function do not change; centralised because they rely on data structures (such as similarity matrices) that must be accessed, and sometimes modified, at each step of the operation; and batch because they run to reach their clustering goal and then stop. Some applications require ongoing processing of massive streams of data. For instance, consider clustering of documents returned by a search engine to extract a topic map [15], or extracting intelligence on possible terrorist attacks from eavesdropping phone calls [29]. This class of applications imposes requirements that classical clustering algorithms do not satisfy [22], such as dynamics in the data stream, and decentralisation for coping with massive data.

In this paper we consider a simplified instance of the data clustering problem. Figure 1 gives a few screen shots of the simulator we developed. Data items are located in a 2D Environment. A possible metric for data clustering is to consider data items initially scattered around randomly, and consider data items on neighbouring coordinates as clustered. Data clustering is accomplished by having agents perform quite simple algorithms, in which agents move data items by picking them up and dropping them next to similar data items. The items are picked up and dropped with respect to some probability which is determined by the presence of similar data items in the local neighbourhood. As such, clusters are formed of data items based on their similarity. We refer to [4] for more details on the specific algorithm, the details of which are of marginal importance for the current paper. Nor do we want to discuss the qualities and deficiencies of the algorithm here. For illustrating our research, we only need $a$ decentralised algorithm that constitutes a self-organising emergent solution, which we can then analyse.

The particular set-up of the data clustering problem that is used for experiments is a situation where in fact, all data is supposed to belong to one cluster. We consider a $2 \mathrm{D}$ environment of size 100x100 units, 200 agents positioned randomly in this environment, not holding an item, and with a view range of 8 units. 1000 data items are randomly scattered in the environment, and a set of neighbouring data items are only considered a cluster if there is a minimum of 3 data items in the cluster. 


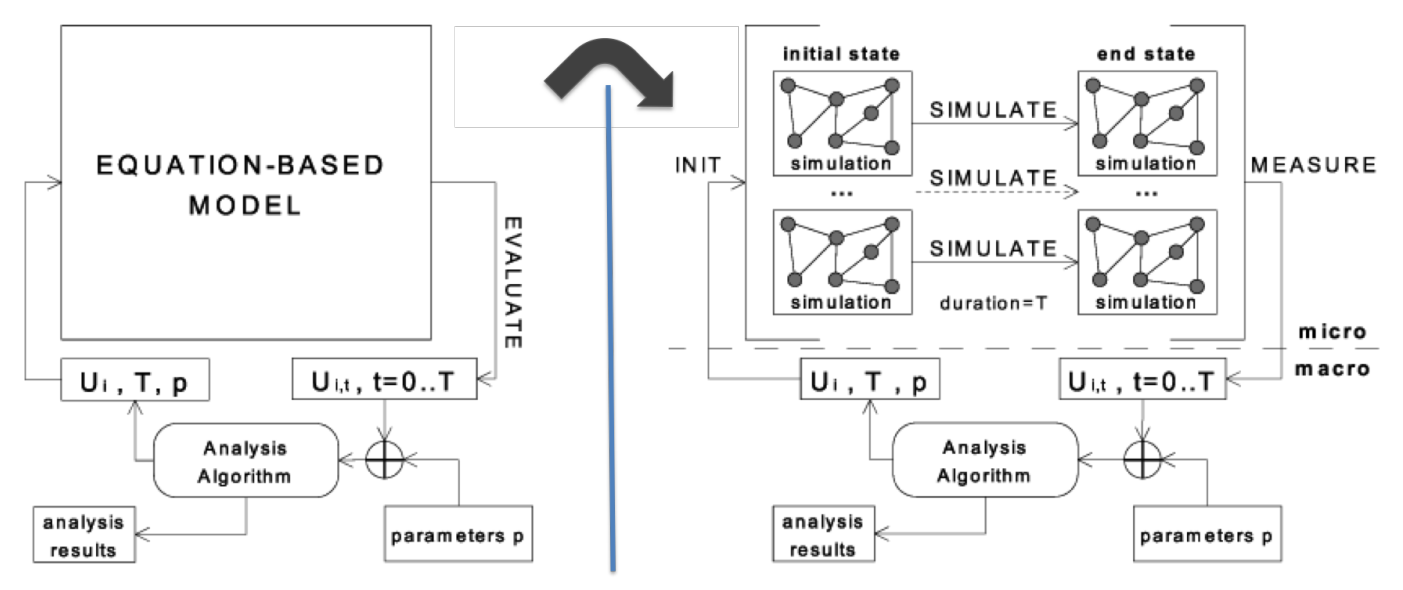

Figure 2. Equation-Free Macroscopic Analysis

Using a self-organising emergent solution has advantages due to the inherent dynamics of the problem (new data items at every moment, agents failing), the inherent decentralisation of the information needed by the agents (location of similar items), and the need for autonomously clustering the stream of data and maintaining the clustered structure. Self-organising emergent solutions have been applied to data mining before $[22,21,23]$. The main problem is that little is known about the exact relation between the microscopic activities/interactions of the agents and the macroscopic clusters that emerge as a result.

\section{Equation-Free Macroscopic Analysis}

In any self-organising emergent system, there are two scales (or levels of description) at which the system can be modelled. On the one hand, the system is fully characterised by the behaviour and interactions between the individual entities; we call this a microscopic description. Mathematically, a microscopic model can be written as

$$
\partial_{t} u=f(u),
$$

in which $\partial_{t}$ represents a time derivative, $u$ represents the full microscopic state of the system (the combined state of all entities, as well as the environment) and $f$ is some evolution law. On the other hand, the system-wide behaviour is more conveniently characterised at the system level, to which we will refer as the macroscopic description. For the data clustering problem, one could, for instance, be interested in observing macroscopic quantities such as the number of clusters and their size. If we could derive a mathematical model for the macroscopic behaviour analytically from the microscopic model (1), which we denote by

$$
\partial_{t} U=F(U),
$$

with $U$ the macroscopic variables, we would be able to fully understand the self-organising emergent behaviour of the system using a wide range of standard mathematical tools.

However, such macroscopic models can only be constructed for relatively simple model problems [28], and simplifications made in order to obtain a macroscopic model for more complex problems can result in significant deviations between the macroscopic model and reality [31]. Therefore, in general cases, a separation in scales prevails between the level of description (the behaviour of the individual entities) and the level of interest for the modeller (the system-wide behaviour), and only a model at the microscopic level is available. For cases in which a macroscopic model of the form (2) cannot be obtained in closed form (although it exists conceptually by definition), a new and promising, equation-free approach has been put forward, based on ideas from numerical analysis $[19,18]$. The key idea in this approach is the construction of a so-called coarse time-stepper, which performs a time step of an unavailable macroscopic model as a three-step procedure: (1) lift (or init), i.e. starting from the macroscopic variables that describe the system-wide behaviour, initialise the full microscopic system in a consistent way; (2) run (or simulate), i.e. perform a simulation with the full microscopic system over a given time interval; and (3) restrict (or measure), i.e. compute the macroscopic state from the full microscopic state of the system. The rationale behind this procedure is the following: if a macroscopic model should conceptually exist, the macroscopic variables by themselves should determine the macroscopic evolution of the system completely, regardless of the specific initial conditions for the remaining degrees of freedom.

Figure 2 shows the analogy between a traditional numerical algorithm, when a macroscopic equation is explicitly available (left), and an equation-free approach (right). If 
(2) would be known explicitly, a set of computational algorithms is available that use this equation to analyse the behaviour of the system. The algorithm iteratively evaluates the equation-based model to obtain the macroscopic values at certain time steps and determines the initial macroscopic states $U_{i}$, the number of evaluations $T$, and some parameter values $p$ for each iteration $i$. The algorithm analyses the obtained results to determine characteristics of the system dynamics. For example, in a time integration routine, we evaluate the time derivative at a given state $U_{i}$ from (2), and make a prediction of the state $U$ at a future time by extrapolation in the obtained direction. That prediction is then the input for a next coarse time step $i+1$, i.e. $U_{i+1}$. When one is interested in the steady state behaviour, one can also directly solve the steady state equation $F(U)=0$; the Newton algorithm will then iteratively evaluate the residual $F\left(U^{(k)}\right)$ and compute a correction.

In the equation-free context (Fig. 2, right), every evaluation of $F(U)$ is replaced by a call to the coarse time-stepper. The microscopic system is initialised from the given values $\left(U_{i}\right)$ of the macroscopic variables (lifting, init in Fig. 2). During the microscopic simulation (simulate), the macroscopic values $U(t)$ are measured at time steps $t$ from the microscopic simulation state $u(t)$ (restriction, measure). We can then perform the numerical algorithms as if we had an explicit formula for the macroscopic model.

Passing information between the microscopic and macroscopic scales at the beginning and end of each iteration (via the lifting / initialisation and restriction/measuring operators) is a vitally important issue. Two (closely related) problems need to be addressed: first, one needs to obtain a set of macroscopic variables that completely determine the evolution of the system at the macroscopic level; these are the variables $U$ that would appear in the macroscopic evolution law (2). Second, one needs to define an operator that initialises a full microscopic system consistently with these macroscopic variables. We note that, once these two steps have been taken, the definition of the measure operator is rather straightforward, since it consists of some postprocessing steps to obtain the macroscopic state from a full microscopic state. Constructing an initialisation operator requires that the relation between the microscopic level and macroscopic level is made explicit; we need to understand what macroscopic variables influence the system behaviour, and how a microscopic realisation of the system depends on these macroscopic variables. As such, once the equationfree methodology is successfully applied for a given selforganising emergent solutions, it will provide a valuable tool to quantify the effects of changes at the microscopic level on the macroscopic behaviour.

In application areas where the equation-free approach has been studied before (i.e. physics, biology, chemistry), the set of macroscopic variables that appear in the macro- scopic evolution equation, and how their value can be computed from the full microscopic system state, are known from underlying physical principles. They follow from a separation of time scales in the system. Using the fact that the microscopic state of the system becomes a function of the macroscopic variables on a time scale that is much faster than the evolution of the macroscopic variables, one can prove that an evolution law should exist for the macroscopic variables only. For engineered systems, such underlying principles are generally not present. Therefore, applying the equation-free methodology faces additional challenges. Specifically, we will simultaneously need to develop a procedure to extract the macroscopic variables reliably from a microscopic simulation, as well as a procedure to initialise the given microscopic system correspondingly.

\section{Defining Macroscopic Variables and Con- structing an Initialisation Operator}

In general, when one designs a self-organising emergent solution, one has a rough idea about the macroscopic variables that should be of interest. In the data clustering example, for instance, the number of clusters, their average size, and the average distance between clusters, can be assumed beforehand to have a big impact on the evolution of the system. It is, however, easy to check that, if we initialise the full microscopic system multiple times with the same values for these variables (but varying the remaining degrees of freedom), the system will not necessarily behave identically, even on the macroscopic level. Consider as an illustrative example the case where there are only two clusters that together contain all data items, which are relatively far away from each other. When one cluster is large and the other one small, the system will quickly evolve to a macroscopic state with only one cluster; if both clusters, however, approximately have the same size, then both clusters will continue to exist over a very long time interval.

Based on this illustration, we can immediately put forward two important observations: (1) a set of macroscopic variables that by themselves uniquely determine the macroscopic evolution of the system is non-trivial to obtain via intuition only; and (2) in general, it is hard to obtain a good quantitative description of the macroscopic behaviour of only one realisation of the system. We will therefore, in the remainder of this text, follow an iterative approach to obtain a complete (and minimal) set of variables that capture the expected macroscopic behaviour when averaged over an ensemble of microscopic realisations. Note that both of these observations deviate from the situation in typical systems that arise in nature, where the equation-free approach has been extensively applied; in those systems, one has theory, based on statistics and separation of time scales, that dictates to a large extent which macroscopic variables to ex- 


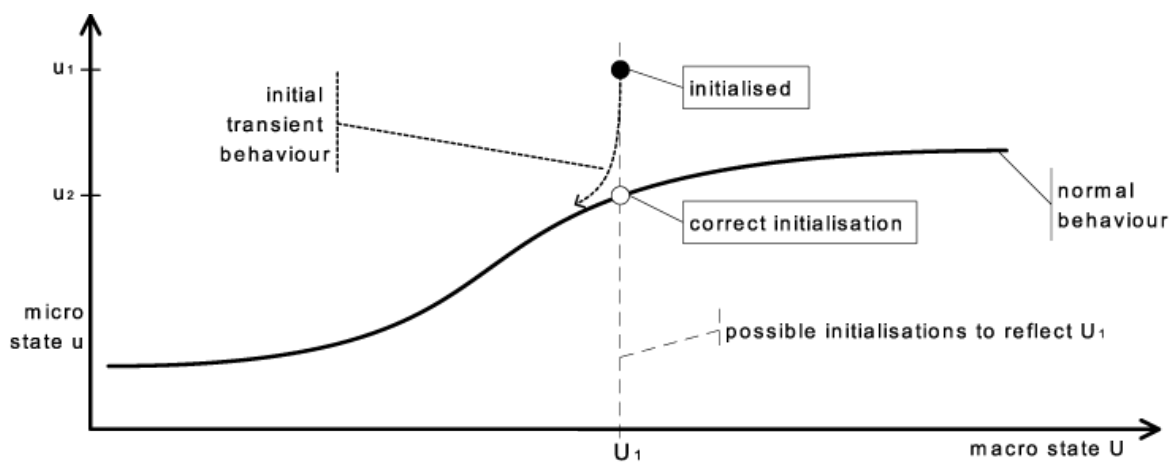

Figure 3. Effects of reinitialisation

pect. Moreover, in most such systems, the number of microscopic entities (molecules, particles) is so large (e.g. Avogadro's number) that averaging over all microscopic entities within one realisation already gives deterministic macroscopic behaviour.

To obtain a valid set of macroscopic variables, we propose a bottom-up approach. Starting from the full microscopic state, we successively aggregate states into a set of more macroscopic variables. In every iteration, we check if the set of aggregated variables is sufficient to determine the evolution of the system. This is done by comparing a long reference simulation with a simulation that is periodically reinitialised, retaining only the values of the aggregated variables and randomising the remaining degrees of freedom. If the aggregated state is an accurate representation of the full microscopic system, such a periodic reinitialisation should not significantly affect the overall macroscopic evolution. The procedure is repeated until we cannot further aggregate the states; the final iteration has then yielded the macroscopic variables.

We illustrate the effects of reinitialisation in Fig. 3. Consider a macroscopic state $U_{1}$; for this macroscopic state, multiple microscopic states $u$ are possible (vertical dashed line). During a normal evolution of the full system, the full microscopic state of the system corresponding to the macroscopic state is represented by the solid line; for the macroscopic variable $U_{1}$, the "correct" microscopic state would be $u_{2}$. However, since the additional degrees of freedom are initialised randomly, the microscopic state after reinitialisation is perturbed, say to $u_{1}$. Then, the microscopic state will evolve quickly towards the solid line. If this would not be true, the system would not be well described by the macroscopic variables. This effect is called healing in the equation-free literature, and it determines how long one should simulate between reinitialisations [19].

\section{Application to the Data Clustering System}

The first step in applying the equation-free methodology is to obtain a clear idea on what we will consider to be the macroscopic behaviour. For the data clustering algorithm, this certainly involves the number of clusters. If we observe a single simulation, starting from randomly positioned agents and data items, we first see a rapid increase in the number of clusters (because initially there are no clusters, while quickly, nearby items will be slightly moved to form small clusters), after which the number of clusters decreases, see Fig. 4. When discussing the macroscopic be-

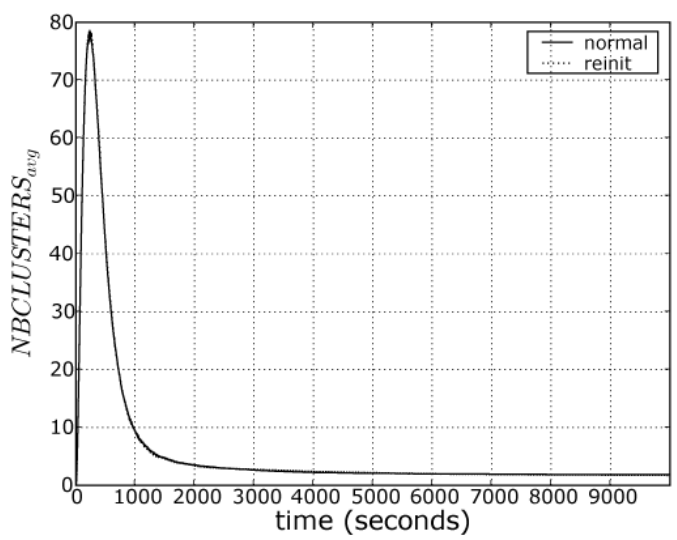

Figure 4. Evolution of the number of clusters, averaged over 100 simulations from random initial conditions.

haviour, we always discard the initial transient, i.e. the first 2000 time-steps. For each individual simulation, the time instances at which clusters vanish appears to be stochastic; however, this randomness disappears if we perform a large number of replica simulations and average the number of clusters over all replicas. We then get a smooth evolution, 


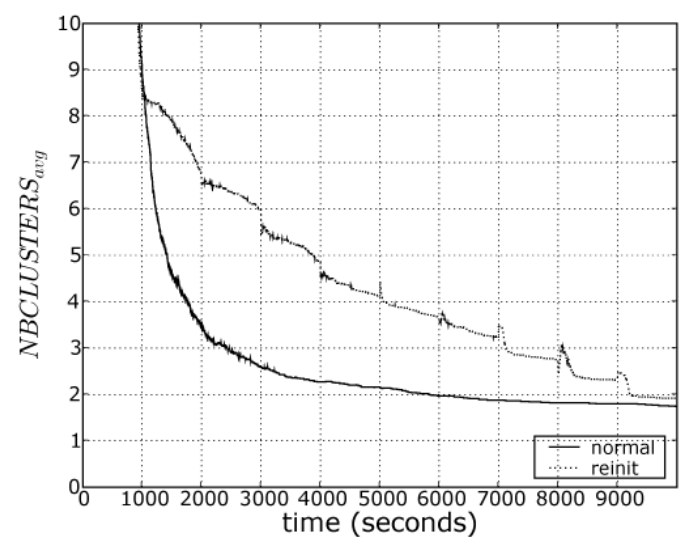

(a) Average number of clusters

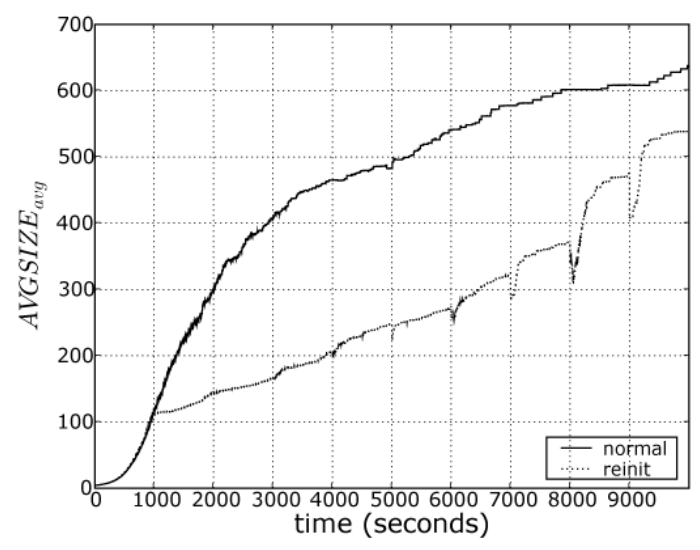

(b) Average cluster size

\section{Figure 5. Comparison of a reference simulation (solid line) and a simulation with reinitialisation after every 1000 time-steps (dashed), with each cluster size set to the average cluster size.}

see Fig. 4. Our simulations indicate that the average number of clusters after a long simulation stagnates at roughly 1.6; our data clustering algorithm therefore does not always reduce the number of clusters to 1 .

We will show how the equation-free approach can be used to systematically study this emerging macroscopic behaviour, which in turn will allow to improve the selforganising emergent solution by improving the microscopic behaviour, yielding better accuracy in the resulting number of clusters.

The microscopic state consists, for each of the replica simulations, of the positions of all data items and agents, the direction of motion of the agents, and which agents are holding data. In a first aggregation, we reinitialise the system using, for each replica simulation, only the position of the centre of the cluster and its size (measured by the number of data items). For now, we also count the number of agents holding a data item (but not which agents). The reinitialisation is performed as follows: for each cluster, we compute its size by counting the number of data items, and its centre as the average position of the data items. We then create new clusters at the computed position with the same number of data items; the exact positions of the data items, however, are randomised. Note that this leaves an indeterminacy in the shape of the cluster. Based on observations of the microscopic state, we found that we need to create roughly circle-shaped clusters, in which $10 \%$ of the positions are vacant. The remaining (unclustered) data items are positioned randomly, but ensuring that all neighbouring positions are vacant. The agents that are holding data items are positioned together with an unclustered data item; the remaining agents are positioned randomly. We verified that a periodic reinitialisation (after every 1000 time-steps) using the above procedure did not alter the macroscopic evolution of the average number of clusters. We therefore conclude that all macroscopic behaviour is still contained in the current aggregated state, and proceed.

As a next step, we discard knowledge about the positions of the individual clusters. To this end, we compute the number of clusters and the average distance between clusters in each replica simulation. Subsequently, we generate new positions for the clusters in the corresponding replica, using only knowledge of this average distance. This is done by positioning the clusters on a regular polygon, of which we also use the centre. At this point, we still retain the individual cluster sizes, and generate new clusters with exactly the same size. We have, however, eliminated the number of agents that is holding a data item as a macroscopic variable; instead, we simply position all agents on random positions and never let them hold a data item. Again, performing a simulation with periodic reinitialisations after every 1000 time-steps showed that the current aggregated state contains all relevant information to retain the macroscopic evolution.

In a following iteration, we try the same with the sizes of the individual clusters. For each replica simulation, we compute the average cluster size. Then, every time we generate a new cluster, the average size is used instead of the cluster size before reinitialisation. We again perform a simulation that is reinitialised after every 1000 time-steps. The results are shown in Fig. 5. We see that the macroscopic behaviour now significantly deviates when reinitialising every 1000 time-steps, and conclude that we also need to take the variation of the cluster sizes into account. Figure 6 shows that the variance of the cluster sizes is eliminated during 


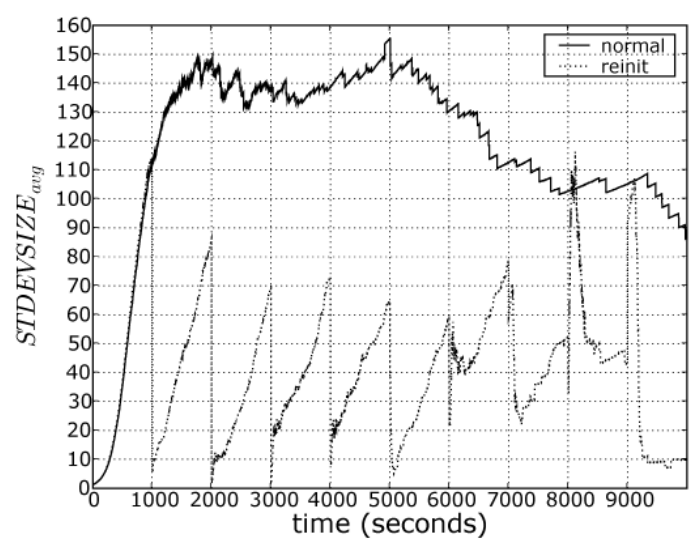

Figure 6 . Standard deviation of the cluster
sizes in each replica simulation, averaged
over all replicas.

each reinitalisation and the simulation evolves as to restore this variance, resulting in artefacts on the evolution of the number of clusters and their average size.

We therefore compute, for each replica simulation, not only the average size, but also the standard deviation, and generate cluster sizes randomly, but such that both quantities are preserved during reinitialisation; see [4] for details. One important additional requirement is that we do not allow arbitrarily small clusters to be created, since they would directly be removed by the clustering algorithm, and therefore do not exist in the reference simulation either. Using this procedure, we again obtain a good agreement between the reference and reinitialised simulations, see Fig. 7.

We subsequently tried performing a reinitialisation without taking the average distance between clusters into account; however, this also produces artefacts.

Up to now, we have reinitialised every replica simulation using the average cluster size (and its standard deviation) and the average distance between clusters. However, the observed macroscopic behaviour of interest is on the average of these quantities over a large number of replica simulations. So, to aggregate our current state once more, we should decide on the number of clusters, the average cluster size (and standard deviation) and the average distance between clusters for each replica simulation, based on the averages of these quantities over all replica simulations. As a first step, we just count the number of clusters in each replica simulation. Then we compute the average cluster size (and standard deviation), where we average over all replica simulations with the same number of clusters. We notice that, for fixed number of clusters, the average cluster size of each replica simulation is roughly the same; there are, however, large differences in the standard deviation.
In fact, the standard deviation of cluster sizes is normally distributed, with a variance that depends on the number of clusters; see Fig. 8. If there are only two clusters, we observe an exponential distribution. (The histograms were obtained using 2300 replicas.) Similarly, the average distance between clusters is also normally distributed over the replicas. We then proceed to aggregate the variables further. We first assign a number of clusters to each replica simulation. Then for each replica simulation, the average cluster size is set to the appropriate average, and a standard deviation, as well as an average distance, are chosen from the appropriate distribution. The resulting procedure is subject to a large number of technical details, see [4]. Once the parameters for each individual replica are known, we proceed with the routines from the previous iteration to initialise each replica. We again verified that this procedure correctly captures the macroscopic behaviour using periodically reinitialised simulations.

Following iterations could try to use only the average number of clusters and the associated standard deviation to generate the number of clusters for each replica simulation. However, we noticed that during this last iteration, we have already reached a scale at which it becomes difficult to draw immediate design conclusions on the effect of the microscopic interaction rules on the macroscopic behaviour. We therefore did not iterate any further.

\section{Discussion}

To date, there is no clear roadmap that allows to directly design microscopic interaction rules to obtain pre-specified macroscopic behaviour. The presented equation-free approach aims to be a step in this direction; it offers an iterative procedure to systematically study the macroscopic behaviour resulting from the microscopic interactions; this could allow to remedy undesirable behaviour. It is important to realise that the proposed bottom-up procedure is highly application-dependent, since one needs to decide which variables to aggregate, and how, in each iteration. However, as a first guideline, we emphasize the importance of gradual coarsening, in which only one variable is aggregated in each iteration of the procedure. This allows to unambiguously determine the cause and the nature of the differences in evolution that are induced by the aggregation of a specific variable.

For the data clustering example, the procedure revealed a number of interesting mechanisms that are active in the proposed solutions. In the first iteration, we found that the algorithm generates circle-shaped clusters, in which $10 \%$ of the positions are vacant. When we reinitialised using only the cluster sizes, but without these vacant places, the average number of clusters decreased more slowly, indicating that the presence of vacant positions is a driving force for clus- 


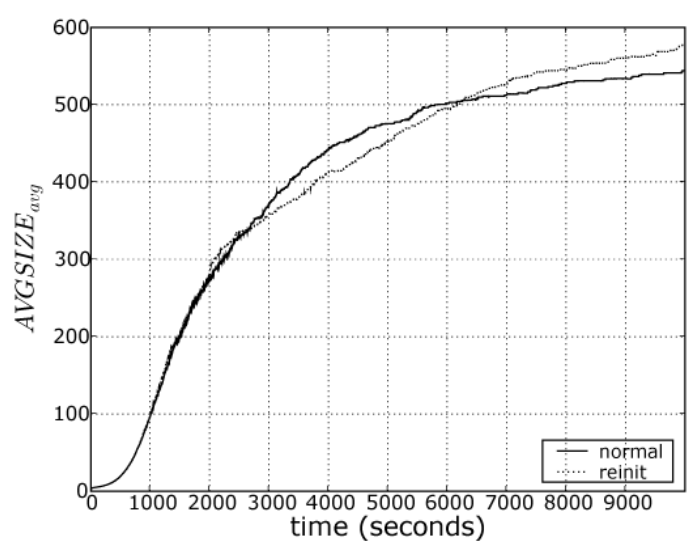

(a) Average cluster size

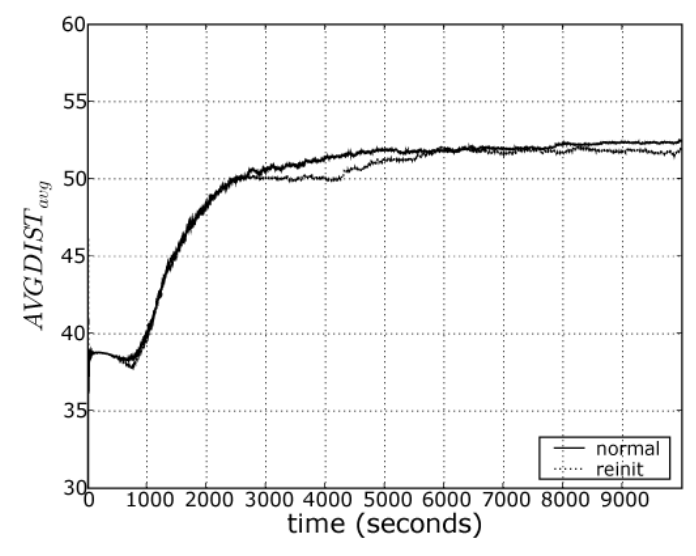

(b) Average distance between clusters

Figure 7. Reinitialisation with average cluster size and standard deviation.

tering. During the second iteration, we observed that both a variation in the size of the clusters, and the average distance between clusters are quantities that influence macroscopic evolution; the number of clusters decreases more rapidly if there is a large difference in size, and if the clusters are closer to each other. These observations can be exploited to improve the microscopic interaction rules. For example, we could make agents aware of the locations of the clusters by putting pheromones in the environment [3]. With a similar mechanism, agents could be stimulated to avoid making multiple clusters of equal (and large) size.

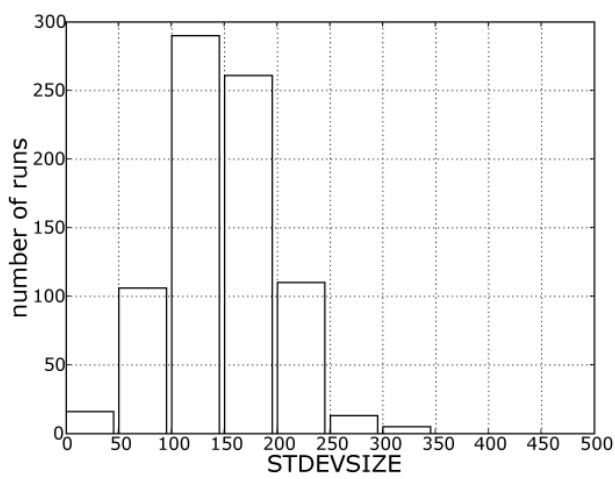
Figure 8. Histogram of the standard deviation
of cluster sizes for 4 clusters, at $t=2000$.

A few warnings are in order when applying this technique. As became clear in the final iterations, as we approach the macroscopic scale, designing an initialisation operator that correctly restores the underlying level, based on only the aggregated state, becomes increasingly in- volved. Therefore, some trade-off needs to be made; one has to realise that the ultimate goal of the procedure is the design of a good self-organising emergent solution. In this context, developing a good quantitative understanding of the macroscopic behaviour of the system is only a tool. In our example, we observed at some point that the advantages of further aggregation would not justify the additional effort. At that point, one should start using the obtained information to enhance the performance at the microscopic level. We defer a discussion of such improvements to a future publication.

Moreover, the procedure requires a large number of simulations. We note, however, that most simulations are done to verify the behaviour after reinitialisation, so this cannot be compared with brute force direct simulations. An important parameter in these simulations is the time interval between reinitialisations, which should be as short as possible (for efficiency), but long enough to eliminate artefacts that are introduced during each reinitialisation.

\section{Related Work}

Equation-free methods have been developed to study systems in a number of scientific fields, in which a macroscopic mathematical model cannot be derived analytically from an underlying microscopic model $[24,19,17]$. A typical example is the simulation of the evolution of the density of bacteria, given only the interactions between individual bacteria [10]. Besides applications, most research has focused on the construction of accurate initialisation operators, which are able to reconstruct the full microscopic state corresponding to a given macroscopic state using only the microscopic simulator [14]. A number of convergence studies have appeared for simple model problems [12, 26, 25]; 
however, for general microscopic problems, some problems still remain [11]. Further algorithmic developments include (so-called projective integration) methods to exploit a separation in time scales to extrapolate the macroscopic states in time (hereby avoiding microscopic simulations over the full time interval)[13, 27].

Initial efforts to apply the equation-free methodology to engineered self-organising emergent software solutions are reported in $[8,7]$, where a projective integration method was used to accelerate simulations of a multi-agent system that describes packet transport using automated guided vehicles; in that work, macroscopic variables were chosen ad-hoc.

To obtain a set of macroscopic variables, based on data from microscopic simulations, the equation-free techniques are being combined with diffusion maps [20], which cluster the data to create a distance measure over the microscopic state space; this measure then gives rise to macroscopic variables that can be used in the equation-free context, see [9] for an example in gene-regulatory networks. We note that, in contrast to the more explicit iterative procedure that we propose, diffusion maps can more easily be viewed as a mathematically founded black box strategy. As such, this technique is not well suited for the problems that we consider here, since we want to study the macroscopic effects of changes to the microscopic rules to engineer a desired macroscopic behaviour.

A different line of related work is the work on software engineering methodologies for self-organising systems. One such methodology is Adelfe. Adelfe is a methodology for developing emergent solutions based on the AMAS theory [2]. In essence, the methodology expects the developer to identify agents and so-called "non-cooperative situations". The expected emergent behaviour is reached by having all agent try to remediate non-cooperative situations. Although interesting as a basic theory, the methodology does not allow to guarantee convergence or stability, or does not provide a disciplined approach to analyse the self-organising system. We expect the equation-free analysis technique to be usable in Adelfe, yet this had not been researched yet. Other methodological approaches focus on providing reusable self-organising emergent solutions as design patterns $[1,5]$. The design patterns can support developers in identifying potential solutions, which then need to be adapted and integrated during the subsequent software development activities. This, however, does not provide any well-founded mathematical foundations for building and analysing self-organising emergent solutions.

\section{Conclusion}

The research that is presented in this paper is in progress, yet we made an important step in the study of equationfree analysis for software systems which lack a mathemat- ical macroscopic model. One conclusion is that the application of equation-free analysis is by far a non-trivial exercise. The iterative application of the technique however proves helpful in thoroughly understanding the link between microscopic and macroscopic behaviour. And if suitable macroscopic variables as well as an initialisation operator are found, a coarse time-stepper can be used to analyse the timely behaviour of the system.

Yet many issues remain unsolved in providing a full approach to engineering self-organising emergent solutions. From the software engineering point of view, appropriate modelling diagrams could assist in specifying the activities and information flow of the microscopic elements, and in deducing or enforcing the presence of feedback loops. For a first step in this direction, we refer to [6]. Also, the effects of incremental changes to the MAS (e.g. due to software evolution) on the variables and initialization operators need to be investigated. Another remaining challenge is to study how our conclusions about adopting equation-free analysis for the data clustering case remain valid for more complex cases, such as the mentioned real-world industrial application of decentralised control of automated guided vehicles in a warehouse environment. Experience in more and more complex cases could allow us to deduce general assumptions on the MAS as prerequisites for adoptability of the approach. Finally, the combination of equation-free analysis with diffusion maps [20] may further improve the efficiency of the technique.

\section{Acknowledgements}

This research is partially funded by the Research Fund K.U.Leuven, the Interuniversity Attraction Poles Programme Belgian State, Belgian Science Policy, and IWT-Flanders. Giovanni Samaey is a Postdoctoral Fellow of the Research Foundation Flanders. Figures are taken from [4].

\section{References}

[1] O. Babaoglu, G. Canright, A. Deutsch, G. A. D. Caro, F. Ducatelle, L. M. Gambardella, N. Ganguly, M. Jelasity, R. Montemanni, A. Montresor, and T. Urnes. Design patterns from biology for distributed computing. ACM Trans. Auton. Adapt. Syst., 1(1):26-66, 2006.

[2] C. Bernon, M.-P. Gleizes, S. Peyruqueou, and G. Picard. ADELFE, a Methodology for Adaptive Multi-Agent Systems Engineering. In P. Petta, R. Tolksdorf, F. Zambonelli, and S. Ossowski, editors, Engineering Societies in the Agents World (ESAW), number 2577 in LNAI, pages 156-169. Springer-Verlag, September 2003.

[3] S. Brueckner. Return from the Ant: Synthetic Ecosystems for Manufacturing Control. PhD thesis, Humboldt University Berlin, 2000. 
[4] T. De Wolf. Analysing and Engineering Self-Organising Emergent Applications. $\mathrm{PhD}$ thesis, Katholieke Universiteit Leuven, May 2007.

[5] T. De Wolf and T. Holvoet. Design patterns for decentralised coordination in self-organising emergent systems. In S. Brueckner, S. Hassas, M. Jelasity, and D. Yamins, editors, ESOA, volume 4335 of Lecture Notes in Computer Science, pages 28-49. Springer, 2006.

[6] T. De Wolf and T. Holvoet. Information Flows for Designing Self-Organising Emergent Systems. In R. Anthony, A. Butler, M. Ibrahim, T. Eymann, and D. J. Veit, editors, Proceedings of the Joint Smart Grid Technologies (SGT) and Engineering Emergence for Autonomic Systems (EEAS) Workshop, pages 22-29, Dublin, Ireland, 2006.

[7] T. De Wolf, T. Holvoet, and G. Samaey. Development of Self-Organising Emergent Applications with Simulationbased Numerical Analysis. In S. A. Brueckner, G. DiMarzo Serugendo, D. Hales, and F. Zambonelli, editors, Engineering Self-Organising Systems: Third International Workshop, ESOA 2005, Utrecht, The Netherlands, July 25, 2005, Revised Selected Papers, volume 3910 of Lecture Notes in Computer Science, pages 138-152. Springer-Verlag, 2006.

[8] T. De Wolf, G. Samaey, T. Holvoet, and D. Roose. Decentralised autonomic computing: Analysing self-organising emergent behaviour using advanced numerical methods. In Proc. of the Second Int'l Conf. on Autonomic Computing, IEEE Computer Society Proc., pages 52-63, 2005.

[9] R. Erban, I. Kevrekidis, D. Adalsteinsson, and T. Elston. Gene regulatory networks: A coarse-grained, equation-free approach to multiscale computation. The Journal of Chemical Physics, 124:084106, 2006.

[10] R. Erban, I. Kevrekidis, and H. Othmer. An equation-free computational approach for extracting population-level behavior from individual-based models of biological dispersal. Physica D: Nonlinear Phenomena, 215:1-24, 2006.

[11] Y. Frederix, G. Samaey, C. Vandekerckhove, T. Li, E. Nies, and D. Roose. Lifting in equation-free methods for molecular dynamics simulations of dense fluids. Technical Report TW-525, Dept. of Computer Science, K.U. Leuven, 2008.

[12] C. Gear, T. Kaper, I. Kevrekidis, and A. Zagaris. Projecting to a slow manifold: Singularly perturbed systems and legacy codes. SIAM Journal on Applied Dynamical Systems, 4(3), 2005.

[13] C. Gear and I. Kevrekidis. Projective methods for stiff differential equations: problems with gaps in their eigenvalue spectrum. SIAM Journal of Scientific Computation, 24(4):1091-1106, 2003.

[14] C. Gear and I. Kevrekidis. Constraint-defined manifolds: a legacy code approach to low-dimensional computation. $J$. Sci. Comp., 2004. In press.

[15] J. Handl and B. Meyer. Improved ant-based clustering and sorting in a document retrieval interface. In Proc. of Parallel Problem Solving from Nature (PPSN VII). Springer, 2002.

[16] A. K. Jain, M. N. Murty, and P. J. Flynn. Data clustering: a review. ACM Comput. Surv., 31(3):264-323, 1999.

[17] I. G. Kevrekidis. Equation-Free Modeling for Complex Systems. online, 2004. (available at http://www.nae.edu/nae/NAEFOE.nsf/weblinks/JHAY65GKQW).
[18] I. G. Kevrekidis, C. W. Gear, and G. Hummer. Equationfree: The computer-aided analysis of complex multiscale systems. AIChE J., 50(7):1346 - 1355, 2004.

[19] I. G. Kevrekidis, C. W. Gear, J. M. Hyman, P. G. Kevrekidis, O. Runborg, and C. Theodoropoulos. Equation-free, coarsegrained multiscale computation: enabling microscopic simulators to perform system-level analysis. Communications in Mathematical Sciences, 1(4):715 - 762, December 2003. (available online at http://www.intlpress.com/CMS/).

[20] B. Nadler, S. Lafon, R. Coifman, and I. Kevrekidis. Diffusion maps, spectral clustering and reaction coordinates of dynamical systems. Appl. Comput. Harmon. Anal, 21:113127, 2006.

[21] R. Parpinelli, H. Lopes, and A. Freitas. Data mining with an ant colony optimization algorithm. IEEE Transactions on Evolutionary Computation, 6(4):321-332, 2002.

[22] H. V. D. Parunak, R. Rohwer, T. Belding, and S. Brueckner. Dynamic decentralized any-time hierarchical clustering. In S. Brueckner, S. Hassas, M. Jelasity, and D. Yamins, editors, Engineering Self-Organising Systems - ESOA 2006, Revised and Invited Papers, volume 4335 of Lecture Notes in Computer Science, pages 66-81. Springer, 2007.

[23] V. Ramos, F. Muge, and P. Pina. Self-organized data and image retrieval as a consequence of inter-dynamic synergistic relationships in artificial ant colonies. In Soft Computing Systems - Design, Management and Applications, 2nd Int. Conf. on Hybrid Intelligent Systems, volume 87 of Frontiers in AI and Applications, pages 500-509. IOS Press, 2002.

[24] C. Theodoropoulos, Y.-H. Qian, and I. G. Kevrekidis. "coarse" stability and bifurcation analysis using timesteppers: A reaction-diffusion example. Proceedings of the National Academy of Sciences, 97:9840-9843, 2000.

[25] P. Van Leemput, W. Vanroose, and D. Roose. Initialization of a lattice boltzmann model with constrained runs. Journal of Computational Physics, 2005. Submitted.

[26] C. Vandekerckhove, I. Kevrekidis, and D. Roose. An efficient Newton-Krylov implementation of the constrained runs scheme for initializing on a slow manifold. Technical report, K.U. Leuven, Dept. of Computer Science, 2007. Submitted for publication.

[27] C. Vandekerckhove, D. Roose, and K. Lust. Numerical stability analysis of an acceleration scheme for step size constrained time integrators. Journal on Computational and Applied Mathematics, 200(2):761-777, 2007.

[28] P. Wegner. Why Interaction is More Powerful than Algorithms. Communications of the ACM, 40(5):80-91, May 1997.

[29] P. Weinstein, H. V. D. Parunak, P. Chiusano, and S. Brueckner. Agents swarming in semantic spaces to corroborate hypotheses. In Proceedings of the Third International Joint Conference on Autonomous Agents and Multiagent Systems. AAMAS 2004., pages 1488-1489, 2004.

[30] D. Weyns and T. Holvoet. Architectural design of a situated multiagent system for controlling automatic guided vehicles. International Journal on Agent Oriented Software Engineering, 2(1):90-128, 2008.

[31] W. Wilson. Resolving Discrepancies between Deterministic Population Models and Individual-Based Simulations. The American Naturalist, 151(2):116-134, February 1998. 